\title{
Percutaneous ultrasound-guided plugged liver biopsy - a single-centre experience
}

\author{
Soumil Singhal ${ }^{1 A, B, C, D, E, F}$, Pradeep M.D. ${ }^{2 B, D}$, Srikar Inuganti ${ }^{2 B}$, Sandeep Botcha ${ }^{2 B}$, \\ Deepashree Thiruchunapalli Deepashree ${ }^{3 A}$, Mangerira C. Uthappa ${ }^{2 A}$ \\ ${ }^{1}$ New Cross Hospital, Wolverhampton, United Kingdom \\ ${ }^{2}$ Gleneagle Global Hospital, India \\ ${ }^{3}$ Rela Institute of Medical Sciences, India
}

\section{Abstract}

Background: Liver biopsy is a widely used, safe diagnostic tool utilised by clinicians for the histopathological assessment of the liver. Our study aims to report our experience in patients who underwent ultrasound-guided plugged percutaneous liver biopsy in a tertiary care hospital in India.

Material and methods: The Institutional Ethical Review Board approved this retrospective study, and informed consent was obtained from all the patients. A total of 830 liver biopsies were performed between January 2014 and December 2018, of which 782 were plugged percutaneous liver biopsies. The tract was plugged using Gelfoam slurry. Various observations related to the procedures were recorded.

Results: Seven hundred and eighty-two were plugged percutaneous liver biopsies, which were performed during the study period. Of the 782 patients, 163 were male, and 619 were female ( $20.8 \%$ and $79.2 \%$, respectively), with a mean age of $49.6 \pm 2$ years ( 1 month to 86 years). A $100 \%$ technical success rate was seen. No immediate major complications were documented in any of the patients who underwent plugged biopsies. No significant complications were seen in any patient.

Conclusions: Percutaneous liver biopsy is an extensively performed diagnostic tool. We found that ultrasound-guided percutaneous plugged liver biopsy is an easy to perform procedure, which is associated with a lower risk of a bleeding complications.

Key words: liver, biopsy, ultrasound, Gelfoam embolisation.

\section{Introduction}

Liver biopsy is a widely used, safe diagnostic tool used by clinicians for the histopathological assessment of the liver. The procedure is associated with low morbidity $(0.2 \%)$ and mortality $(0.01 \%)$, and hence is considered relatively safe $[1,2]$. The first reported case of a liver biopsy performed for a diagnostic purpose dates back to 1923 [3]. The procedure has evolved over the last 70 years and has become an important tool in the clinician's armamentarium. A liver biopsy can be performed either via the percutaneous route or via the transjugular route. Severe haemorrhage is the most common complication, which can occur secondarily to a hepatic artery or portal vein injury. Altered coagulation status is prevalent in patients with liver disease, increasing the risk of post-procedure bleeding complications. McGill et al., in a study comprising 9212 liver biopsies, found that $0.11 \%$ of patients presented with fatal procedure-related bleeding complications and $0.24 \%$ presented with non-fatal bleeding. In patients with ascites and coagulopathy, transjugular liver biopsy has been found be a safer option [4].

Correspondence address:

Dr. Soumil Singhal, New Cross Hospital, Wolverhampton, United Kingdom, e-mail: drsoumilsinghal75@gmail.com

Authors' contribution:

A Study design · B Data collection · C Statistical analysis · D Data interpretation · E Manuscript preparation · F Literature search · G Funds collection 
Transjugular liver biopsy (TJLB) is an invasive procedure that requires an internal jugular vein access. Transjugular liver biopsy, when compared to percutaneous liver biopsy, yields small sample sizes with the fragmentation of samples when compared to the samples via the percutaneous route. Because of its simplicity and high yield of tissue samples, percutaneous liver biopsy is the widely preferred procedure. A modification in the procedure was introduced to avoid the potential risk of bleeding, which was referred to as plugged liver biopsy (PLB).

PLB was first described in 1984 as a modification of the standard percutaneous biopsy $[5,6]$. Ultrasoundguided percutaneous plugged liver biopsy is performed in patients with coagulopathy when TJLB is not available. The procedure is performed like any other conventional percutaneous liver biopsy until the sample has been acquired. Following the acquisition of the sample, Gelfoam slurry/Gelfoam torpedo is injected via the co-axial needle.

Gelfoam, which is an embolic material, prevents post-biopsy bleeding through the capsular puncture site. Ultrasound-guided PLB is easy to perform, is less time consuming, and is associated with a lower risk of bleeding [7]. Our study aims to present a tertiary care experience in patients who underwent ultrasound-guided plugged percutaneous liver biopsy.

\section{Material and methods}

A retrospective study was performed in 830 patients who underwent liver biopsy in the Intervention Radiology Department of our institution (Gleneagles Global Hospital-Bangalore \& Chennai) between January 2014 and December 2018. Approval from the Institutional Review Board was obtained. The patients were identified using the hospital's RIS (Radiology Information System), and their details were obtained. Details of the clinical indications of the procedure, histopathological reports, and post-procedural events, including complications, were reviewed in our study.

\section{Patient preparation and diagnostic workup}

All our patients were given an explanation about liver biopsy in detail, its indications, its associated complications, and alternative options, following which informed consent was obtained. Initial pre-procedural workup, which includes various laboratory investigations such as complete blood count (CBC), prothrombin time (PT), international normalized ratio (INR), and serology, were performed and reviewed before the procedure. All prior radiological investigations conducted were reviewed. Screening ultrasound was performed in all patients to plan the procedure. Patients on aspirin continued to take their medication. Clopidogrel was withheld for 5 days before the procedure. Patients who were on dual platelets were asked to discontinue clopidogrel for 5 days but con- tinued to take their aspirin as usual. Patients who were on low molecular weight heparin were asked to withhold one dose before the procedure. Warfarin was withheld for 5 days and the patient was started on low-molecularweight heparin. A correction was given in patients when the INR value was more than 1.5 and the platelet count was less than 50,000 platelets per microlitre of blood. Patients with deranged INR were transfused with 10 to $15 \mathrm{ml} / \mathrm{kg}$ of fresh frozen plasma immediately prior to the procedure. Patients were transfused either a unit of single donor platelet or about 4 units of random donor platelets for correction.

\section{Screening ultrasound}

All screening ultrasound was performed on a GE LOGIQ 7 (Waukesha, WI, US) or Mindray M7 (Shenzhen Mindray Biomedical Electronics Co. Ltd., Shenzhen, China) ultrasound machine using a 1-5 MHZ convex transducer to evaluate the biopsy site. The possibility of any anatomical variation (for example, Chilaiditi syndrome, in which a bowel loop lies between the abdominal wall and liver) was ruled out to avoid inadvertent puncture of adjacent viscus. Screening ultrasound also permitted the detection of focal lesions (which may or may not have been previously suspected). Ultrasound scan guidance helps in achieving the best biopsy sample rate.

\section{Biopsy technique}

The convex transducer is prepared using all sterile aseptic precautions. Initially, local anaesthetization of the skin and subcutaneous tissue was performed by injecting $2 \%$ lignocaine (without adrenaline) using a 26 gauge $(26 \mathrm{G})$ needle. The needle is further advanced under ultrasound guidance anaesthetizing up to the pericapsular region, and infiltration can be seen in the form of indentation of the liver capsule on real-time ultrasound. A small skin incision was made. An $18 \mathrm{G} \times 10 / 16 \mathrm{~cm}$ BARD co-axial (Mission semi-automatic system, Tempe, AZ) and Cook (Bloomington, IN, USA) core biopsy instrument was used for the procedure. The co-axial needle was advanced under ultrasound guidance to avoid any significant vascular or critical structures in the chosen path. Once the tip of the co-axial needle was positioned following the capsular puncture into the region of interest, 3 or 4 cores were acquired. The liver capsule was penetrated only once to avoid the risk of bleeding. The grossly examined sample was carefully transferred into a container filled with formalin. An $18 \mathrm{~mm}$ tissue sample was considered full length when a $20 \mathrm{~mm}$ needle throw was performed.

\section{Plugging of the needle tract}

Following the biopsy, the needle tract was plugged using Gelfoam slurry. Gelatine sponge slurry was prepared by 

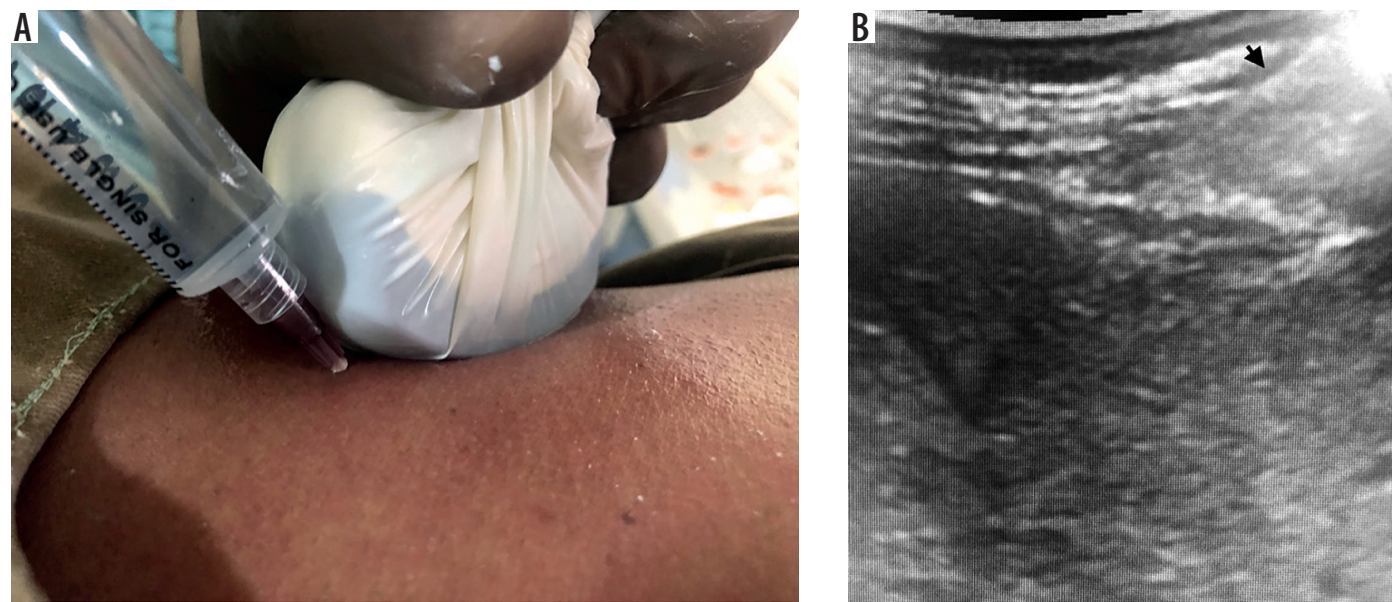

Figure 1. Local anaesthetization of the skin and subcutaneous tissue under ultrasound guidance. Black arrow shows the needle tip on the subcutaneous plane

cutting the sheet into a tiny pledget, which was placed in one of the syringes. The other syringe was filled with $5 \mathrm{ml}$ of normal saline and connected with the first using a 3-way stopcock. Using the Tessari technique, the pledgets were macerated into a thick slurry-like consistency. This temporary embolic preparation was injected into the needle tract via the co-axial needle by slowly withdrawing the sheath up to the capsular surface.

\section{Post-procedure care}

A tight compression bandage is applied at the biopsy site, and the patient is placed in a right decubitus position. Daycare patients are kept under observation for 4 hours, while inpatients are discharged after 1 day of observation. Post-procedural complications, if there are any, are looked for and documented as per the Society of Interventional Radiology guidelines [7]. Some patients complained of mild pain in the right shoulder region post-biopsy, which subsided after taking analgesics.

\section{Results}

During a 5-year period we performed a total of 830 liver biopsies, of which 48 were TJLBs. Hence, a total of 782 plugged percutaneous liver biopsies were performed during the study period. Of the 782 patients, 163 were male and 619 were female ( $20.8 \%$ and $79.2 \%$, respectively) with a mean age of $49.6 \pm 2$ years and a median age of 53 years (1 month to 86 years). Five hundred and fifty $(70.27 \%)$ biopsies were non-targeted while the remaining 232 (29.7\%) were targeted lesion biopsies. Being a large transplant unit, the main indication of a non-targeted liver biopsy was to rule out a diffuse parenchymal disease, whereas targeted lesion biopsies were performed to achieve a definitive diagnosis. Segment VI of the liver was considered as the first site for non-targeted biopsy. Cases with ascites fluid were drained before performing a biopsy. Left segment II was considered in cases when right perihepatic fluid could not be drained completely and there was availability

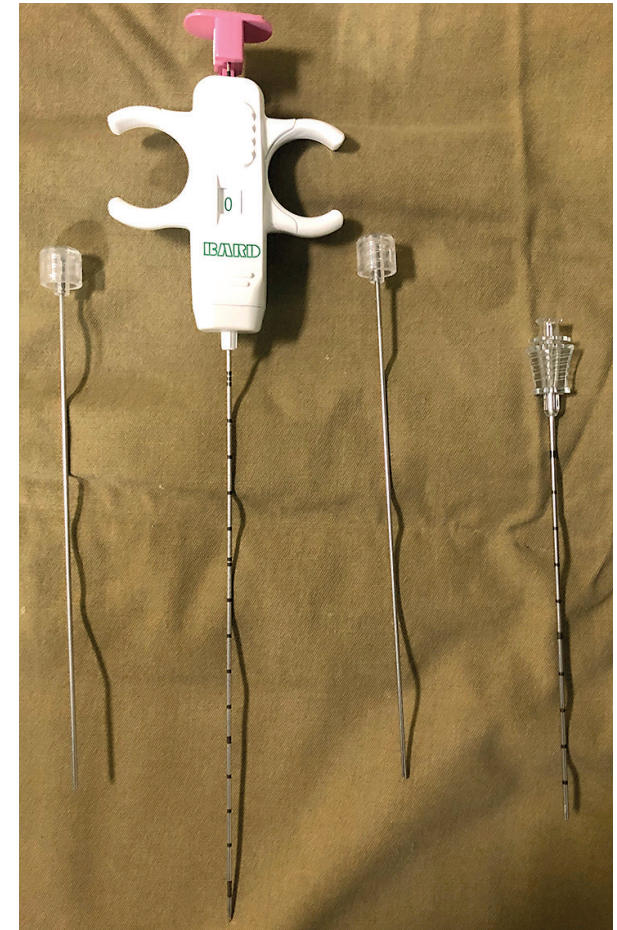

Figure 2. $18 \mathrm{G} \times 16 \mathrm{~cm}$ semi-automatic system BARD co-axial (Mission, Tempe, AZ)

of a safe acoustic window around left segment II. Eightythree per cent of the biopsies were taken from the right lobe of the liver. Targeted biopsy was performed on lesions ranging from 1.0 to $12.8 \mathrm{~cm}$ (mean $3.8 \mathrm{~cm}$ ). Lesions were normally approached by passing through normal liver parenchyma to avoid bleeding complications. In cases of multiple lesions, those without necrosis and those in the right lobes were considered as primary targets. In the case of necrotic lesions, biopsy was mainly performed from the wall for better histopathological yield.

In our cohort we came across several lesions adjacent to large vessels in a technically challenging site; in these cases we either chose another lesion if available or aimed at approaching the lesion on an axis parallel to the vessel. We also used a $20 \mathrm{G}$ needle with a $10 \mathrm{~mm}$ throw to avoid any inadvertent vascular injury. 

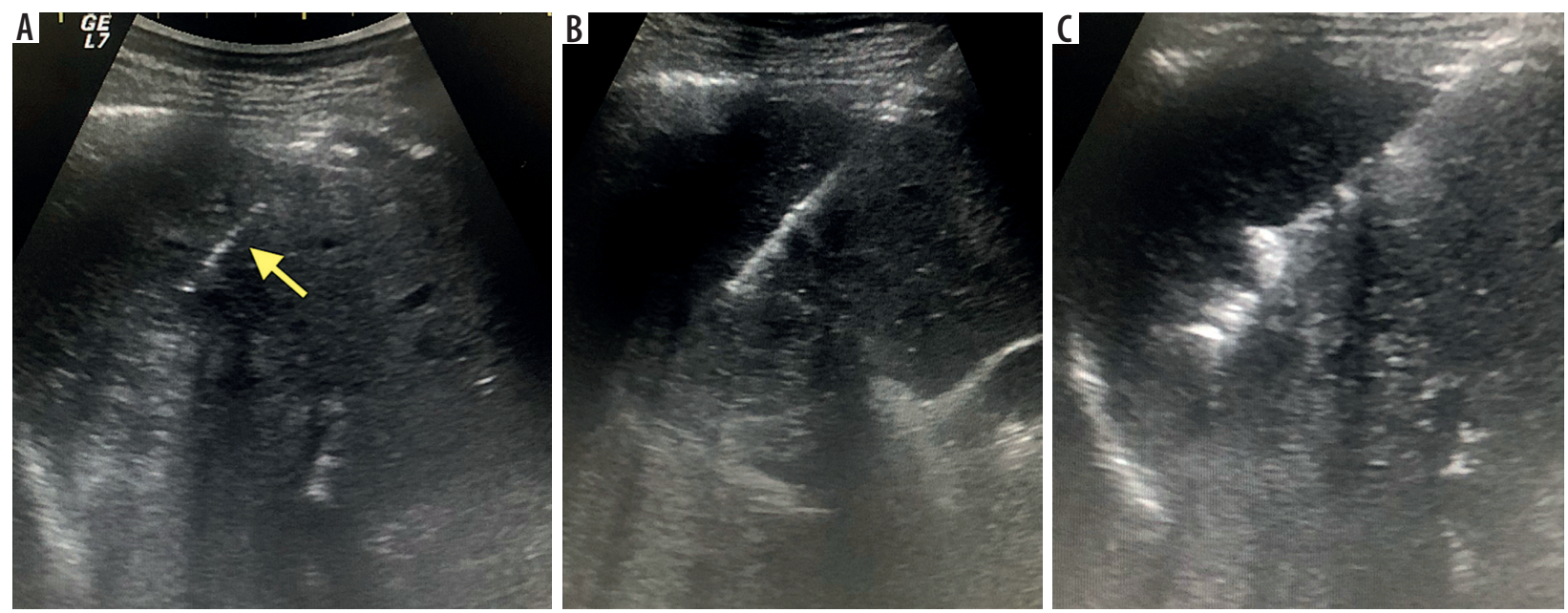

Figure 3. Sequential images showing an ultrasound guided plugged liver biopsy, co-axial needle (yellow arrow) positioned, followed by acquisition of liver tissue and plugging of tract using Gelfoam slurry

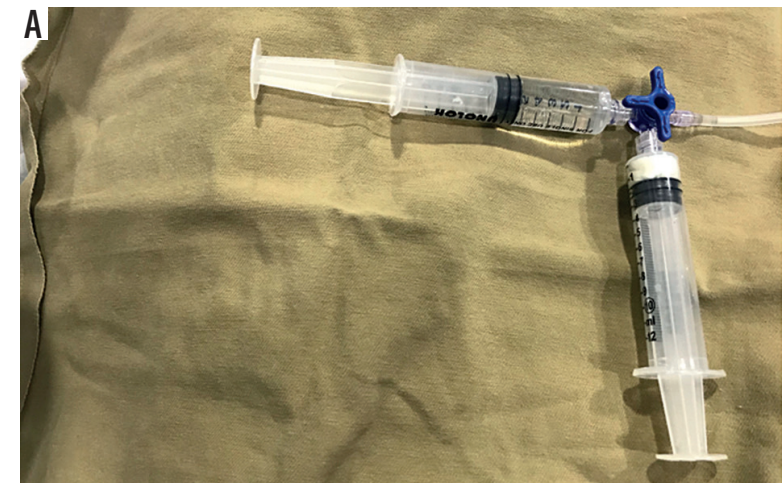

Figure 4. Tessari technique: procedure performed to make Gelfoam slurry

All the biopsies were technically successful in obtaining a histopathological diagnosis because of an adequate sample. The number of needle passes ranged from 1 to 4 , with a mean of $2 \pm 0.7$. Mean sample length was about $18 \mathrm{~mm}$, which included an average of 8.6 portal tracts.

Post procedure, all patients were observed for 4 hours. Patients who developed pain, hypotension, localised tenderness, or abdominal tenderness were re-evaluated with an ultrasound. Any presence of free fluid in the perihepatic region prompted a 24 -hour observation of the patient and a repeat complete blood count. If no fluid was noted, conservative management was implemented. Forty-three per cent of patients reported minor complications in the form of pain or discomfort in the right hypochondriac region or the right shoulder region, which settled on conservative management (including reassurance and using analgesics). After settlement of the patients' complaints, a repeat assessment of vitals was made and patients were discharged with a guideline to immediately attend the casualty department in case of an emergency. Twenty-nine patients demonstrated some free fluid requiring a 24-hour observation; none of them needed any further management. No repeat biopsies were performed in any patient. No immediate major complication was documented in any of the patients who underwent plugged percutaneous

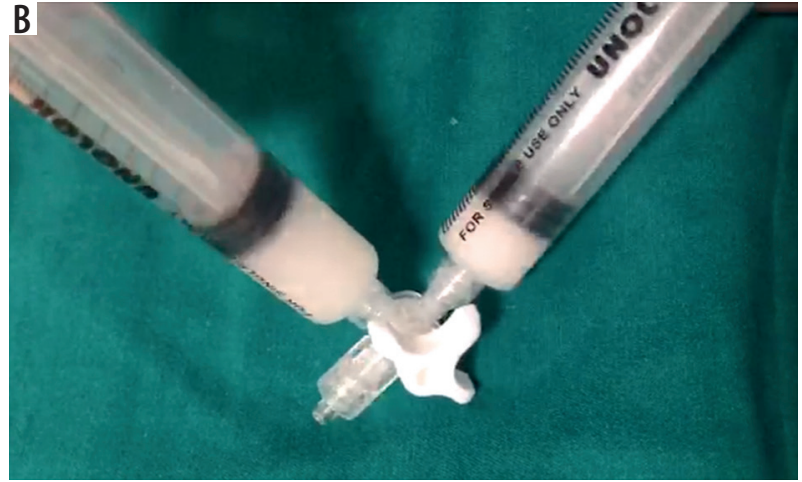

liver biopsies. The technical success of PLB was 100\% in our study.

Our observation during the study revealed a diverse spectrum of patients who were referred for percutaneous liver biopsy. The spectrum included patients who were suspicious of chronic liver disease, patients with bleeding tendencies, for pre-transplant workup, post-liver transplant, and those suspected of primary malignancy or metastasis. Patients with bleeding tendencies were given adequate correction before the procedure. One hundred and thirty-four patients (17.1\%) had INR values over 1.5, and platelet counts less than 50,000 platelets per microlitre of blood, for which correction was given before the procedure.

The largest subgroup of patients in our study was the post-liver transplant group. One hundred and thirty patients (16.6\%) were diagnosed to have graft rejection, and 10 patients $(1.3 \%)$ were diagnosed to have post-transplant hepatitis and sepsis, respectively. Two patients $(0.25 \%)$ had features of tuberculosis, and five $(0.64 \%)$ post-transplant patients had normal liver parenchyma.

During the pre-transplant evaluation, $32(4.1 \%)$ patients showed features of steatosis, and $8(1.02 \%)$ patients showed non-alcoholic steatohepatitis features. Thirteen patients (1.66\%) had normal donor liver parenchyma. 
Table 1. Characteristics of percutaneous plugged liver biopsy procedures

\begin{tabular}{|l|c|}
\hline Total liver biopsy & 782 \\
\hline Male & $163(20.8 \%)$ \\
\hline Female & $619(79.2 \%)$ \\
\hline Targeted biopsy & $29.7 \%$ \\
\hline Non-targeted biopsy & $70.27 \%$ \\
\hline Total number of passes & $1-4(2 \pm 0.7)$ \\
\hline Mean sample length & $18 \mathrm{~mm}$ \\
\hline Average portal tract & 8.6 \\
\hline Minor complications (pain, discomfort) & $43 \%$ \\
\hline
\end{tabular}

Thirteen samples demonstrated features of steatosis. Fibrosis was seen in 99 patients, of whom 5 had noncirrhotic portal fibrosis. One hundred and fifty-two patients showed features of hepatitis, of whom $12(1.53 \%)$ were non-alcoholic steatohepatitis, 13 (1.63\%) were autoimmune hepatitis, and $8(1.02 \%)$ were secondary to hepatitis C. Fifteen patients showed features of chronic liver disease.

Targeted lesion biopsy revealed hepatocellular carcinoma, cholangiocarcinoma, and metastasis in 48 (6.1\%), $40(5.1 \%)$, and $111(14.1 \%)$ patients, respectively. Hodgkin's lymphoma, non-Hodgkin's lymphoma, diffuse B-cell lymphoma, haemangioma, rhabdomyosarcoma, and undifferentiated mesenchymal tumour were seen in 2 patients $(0.25 \%)$ respectively. Other indications for the procedure included a) inflammation, b) Wilson's disease, c) cholestasis, d) infection, and e) obstructive cholangiopathy.

Gelfoam track embolization in our case series was found to be very effective in preventing major complications.

\section{Discussion}

Percutaneous liver biopsy is a gold standard technique used to acquire a tissue sample, which helps in facilitating the diagnosis [6-8]. Haemorrhage is the most common serious complication associated with the procedure. Bleedings are mainly secondary to arterial, portal, or intercostal arteries. A study that included 9212 liver biopsies found that $0.11 \%$ and $0.24 \%$ presented with fatal and non-fatal haemorrhage, respectively [9]. Post-biopsy bleeds are related to 3 factors: 1) patient-related, 2) technical causes, or 3) lesion-associated causes. Patient-related factors include chronic liver disease, coagulopathy, thrombocytopaenia, platelet dysfunction, haematological malignancy, ascites, medications, or non-compliant patient [10-12].

Technical factors affecting the outcome include the number of cores acquired, the size of the needle used, nonguided biopsies, and operator experience [13]. Lesionrelated factors include the location of the lesion, its proximity to major vascular structures, and its hyper-vascular nature. Transjugular liver biopsies have been advocated in the past as a reliable technique in patients with bleeding
Table 2. Indications for percutaneous plugged liver biopsy procedures

\begin{tabular}{|l|c|c|}
\hline Diagnosis & No. of patients & Percentage (\%) \\
\hline Graft rejection & 130 & 16.50 \\
\hline Hepatitis & 119 & 15.20 \\
\hline Metastasis & 111 & 14.10 \\
\hline Fibrosis & 94 & 12.00 \\
\hline Hepatocellular carcinoma & 48 & 6.10 \\
\hline Cholangiocarcinoma & 40 & 5.10 \\
\hline Donor liver steatosis & 32 & 4.10 \\
\hline Inflammatory & 31 & 3.96 \\
\hline Normal liver & 23 & 3.00 \\
\hline Cholestasis & 13 & 1.66 \\
\hline Autoimmune hepatitis & 13 & 1.66 \\
\hline Donor normal liver & 13 & 1.66 \\
\hline NASH (non-alcoholic steatohepatitis) & 12 & 1.53 \\
\hline Cirrhosis & 10 & 1.30 \\
\hline Steatosis & 2 & 1.30 \\
\hline Transplant liver hepatitis & 10 & 1.30 \\
\hline Transplant liver sepsis & 10 & 1.30 \\
\hline Donor liver - NASH (non-alcoholic & 8 & 1.02 \\
\hline steatohepatitis) & 10 & 0.25 \\
\hline Hepatitis C with recurrent fibrosis & 8 & 1.02 \\
\hline Infection & 8 & 1.02 \\
\hline Normal transplant liver & 5 & 0.64 \\
\hline Wilson's disease & 5 & 0.64 \\
\hline NCPF (non-cirrhotic portal fibrosis) & 5 & 0.64 \\
\hline Obstructive cholangiopathy & 5 & 0.64 \\
\hline Chronic liver disease (CLD) & 25 \\
\hline Hodgkin's lymphoma & 25 \\
\hline Non-Hodgkin's lymphoma & 25 \\
\hline Diffuse B-cell lymphoma & 25 \\
\hline Transplant liver tuberculosis & 2 & \\
\hline Haemangioma & 2 & 0.64 \\
\hline Rhabdomyosarcoma & 25 \\
\hline Undifferentiated mesenchymal & 2 & \\
\hline tumour & 2 & \\
\hline
\end{tabular}

diathesis, poor respiration, and massive ascites [14,15]. However, TJLB has several drawbacks, including difficulty in acquiring biopsy from difficult sites (lesions away from a hepatic vein), inadequate sample acquisition, puncture site complications, arrhythmias, and haemoperitoneum [16-19]. TJLB is associated with large inter-operator variability and a steep learning curve, which can affect the outcome of the procedure. A meta-analysis reviewing 7526 TJLBs reported a technical failure rate of 3.2\% [4]. Lebrec et al. reported intra-peritoneal haemorrhage as the most serious complication that occurs secondarily to capsular 
tear [20]. Kalambokis et al. identified major and minor procedure-related complications in $0.2 \%$ and $3 \%$ of patients, respectively [4].

PLB was first described in 1984 by Riley et al. [5] as an alternative to the standard percutaneous biopsy. The procedure involved the post-biopsy embolization of the needle track in reducing the risk of haemorrhage. It is quicker, easier, and less time consuming as compared to TJLB. Gelfoam is most commonly used as an embolic agent. Gelfoam is an absorbable derivative of purified porcine skin. It acts by inducing a thrombus formation as well as providing structural integrity to the thrombus. It is widely used because it is readily available and cheap. Gelfoam is used in the form of either a torpedo or a slurry or both. Complications associated with the procedure include infection, biliary leak, biliary fistula, arterio-portal fistula, major bleeding, and death. Several small sample size studies have been conducted in the past, which have reported an overall success rate of $95 \%$ and a complication rate of less than $2 \%[6,14-16,21,22]$. Sawyer et al. found no serious bleeding complications when PLB was performed in 100 patients with liver disease and coagulopathy [21].

Another study by Kamphuisen $e t$ al. in patients with chronic liver disease with coagulopathy found that PLB was technically successful and complication-free [16]. Our study reported no major post-biopsy haemorrhage; this finding was similar to those reported in the few previous studies $[6,15,16]$.

Graft rejection is a significant concern in the posttransplant liver, with biopsy playing a vital problem-solving role. Either TJLB or PLB can be performed in these cases. Azoulay et al. noted a 13\% technical failure rate when TJLB was performed within 30 days of the post- transplant period [23]. Risk of bleeding is high in the early post-transplant period, mainly due to thrombocytopaenia and ascites $[4,7,23,24]$. Park et al. reported the use of $\mathrm{N}$-butyl cyanoacrylate as an alternative embolic agent for track embolization [25].

According to our literature search, our study is the most extensive series of plugged liver biopsy using Gelfoam to date. We found that PLB was a safe and feasible technique to obtain a diagnosis in any clinical scenario, with a high level of confidence. Our findings are comparable with those of all previously reported studies.

Our study had some limitations, including its retrospective study design; a case-control design would give more strength supporting plugged liver biopsy over conventional percutaneous biopsy. Nevertheless, our study included a spectrum of conditions in which we used PLB over traditional percutaneous liver biopsy. A prospective case-control design is warranted for the same.

\section{Conclusions}

Percutaneous liver biopsy is an extensively performed diagnostic tool used in our everyday practice. However, it is associated with procedure-related bleeding complications. We found that ultrasound-guided percutaneous plugged liver biopsy is an easy to perform, less time-consuming procedure, which is associated with a lower risk of bleeding complications.

\section{Conflict of interest}

The authors report no conflict of interest.

\section{References}

1. Lindner H. Grenzen und Gefahren der perkutanen Leberbiopsie mit der Menghini-Nadel: Erfahrungen bei 80000 Leberbiopsien. Dtsch Med Wochenschr 1967; 92: 1751.

2. Zamcheck N, Klausenstock O. Liver biopsy. The risk of needle biopsy. N Engl J Med 1953; 249: 1062-1069.

3. Bingel A. Ueber die parenchympunktion der leber. Verh Dtsch Ges Inn Med 1923; 35: 210-212.

4. Kalambokis G, Manousou P, Vibhakorn S, et al. Transjugular liver biopsy - indications, adequacy, quality of specimens, and complications - a systematic review. J Hepatol 2007; 47: 284-294.

5. Riley SA, Ellis WR, Irving HC, et al. Percutaneous liver biopsy with plugging of needle track: a safe method for use in patients with impaired coagulation. Lancet 1984; 2: 436.

6. Tobin MV, Gilmore IT. Plugged liver biopsy in patients with impaired coagulation. Dig Dis Sci 1989; 34: 13-15.

7. Gupta S, Wallace MJ, Cardella JF, et al. Quality improvement guidelines for percutaneous needle biopsy. J Vasc Interv Radiol 2010; 21: 969-975.
8. Colonna JO 2nd, Brems JJ, Goldstein LI, et al. The importance of percutaneous liver biopsy in the management of the liver transplant recipient. Transplant Proc 1988; 20: 682-684.

9. McGill DB, Rakela J, Zinsmeister AR, Ott BJ. A 21-year experience with major. hemorrhage after percutaneous liver biopsy. Gastroenterology 1990; 99: 1396-1400.

10. Albeniz Arbizu E, Lopez San Roman A, Garcia Gonzalez M, et al. Fibrin-glue sealed liver biopsy in patients with a liver transplantation or in liver transplantation waiting list: preliminary results. Transplant Proc 2003; 35: 1911-1912.

11. Chuah SY. Liver biopsy - past, present and future. Singapore Med J 1996; 37: 86-90.

12. Sherlock S, Dick R, Van Leeuwen DJ. Liver biopsy today. The Royal Free Hospital Experience. J Hepatol 1984; 1: 75-85.

13. Azar N, Delman T, Nakamoto D. Transcutaneous management of bleeding after solid organ biopsy what the radiologist needs to know and use. US Radiology 2011; 3: 53-56. 
14. Zins M, Vilgrain V, Gayno S, et al. US-guided percutaneous liver biopsy with plugging of the needle track: a prospective study in 72 high-risk patients. Radiology 1992; 184: 841-843.

15. Atar E, Ben Ari Z, Bachar GN, et al. A comparison of transjugular and plugged-percutaneous liver biopsy in patients with contraindications to ordinary percutaneous liver biopsy and an "in-house" protocol for selecting the procedure of choice. Cardiovasc Intervent Radiol 2010; 33: 560-564.

16. Kamphuisen PW, Wiersma TG, Mulder CJ, de Vries RA. Pluggedpercutaneous liver biopsy in patients with impaired coagulation and ascites. Pathophysiol Haemost Thromb 2002; 32: 190-193.

17. Lebrec D, Goldfarb G, Degott C, et al. Transvenous liver biopsy: an experience based on 1000 hepatic tissue samplings with this procedure. Gastroenterology 1982; 83: 338-340.

18. Velt PM, Choy OG, Shimkin PM, Link RJ. Transjugular liver biopsy in high-risk patients with hepatic disease. Radiology 1984; 153: 91-93.

19. Wolska-Krawczyk M, Krawczyk M, Katoh M, et al. Liver fibrosis: how many samples in transjugular liver biopsy are sufficient? Histological vs. clinical value. Abdom Imaging 2013; 38: 461-464.
20. Lebrec D, Goldfarb G, Degott C, et al. Transvenous liver biopsy: an experience based on 1000 hepatic tissue samplings with this procedure. Gastroenterology 1982; 83: 338-340.

21. Sawyerr AM, McCormick PA, Tennyson GS, et al. A comparison of transjugular and plugged percutaneous liver biopsy in patients with impaired coagulation. J Hepatol 1993; 17: 81-85.

22. Smith TP, McDermott VG, Ayoub DM, et al. Percutaneous transhepatic liver biopsy with tract embolization. Radiology 1996; 198 : 769-774.

23. Azoulay D, Raccuia JS, Roche B, et al. The value of early transjugular liver biopsy after liver transplantation. Transplantation 1996; 61: 406-409.

24. Van Ha TG. Liver biopsy in liver transplant recipients. Semin Intervent Radiol 2004; 21: 271-274.

25. Park SY, Kim J, Kim BW, et al. Embolization of percutaneous transhepatic portal venous access tract with $\mathrm{N}$-butyl cyanoacrylate. $\mathrm{Br}$ J Radiol 2014; 87: 20140347. 